\title{
Impact of Telemedicine on Hospitalisation and Mortality Rates in Community- Based Haemodialysis Centres in Singapore During the COVID-19 Pandemic
}

Tripti $\underline{\text { Singh, }},{ }^{1,2} M B B S, M R C P, F A M S$, Clara LY $\underline{\text { Ngoh }},{ }^{2} M b C h B, M R C P, F A M S$, Weng Kin Wong, ${ }^{2} M B B S, M R C P, F A M S$, Behram Ali Khan, ${ }^{1,2}$ MBBS, MD, ABIM (Neph/IM), FAMS

\begin{abstract}
Introduction: With the unprecedented challenges imposed on the modern healthcare system due to the COVID-19 pandemic, innovative solutions needed to be swiftly implemented to maintain clinical oversight on patient care. Telemedicine was introduced in Singapore in community-based haemodialysis (HD) centres to comply with the Ministry of Health's directives on movement restriction of healthcare workers and related measures to minimise the spread of SARS-CoV-2 in healthcare facilities.

Methods: We describe here our experience of 26 community haemodialysis centres in Singapore, analysing clinical audit data, as well as comparing hospitalisation and mortality rates as outcomes in the time frames of pre- and post-introduction of telemedicine.

Results: We found that the hospitalisation rate was $13.9 \%$ (95\% CI: $5.6 \%-21.5 \%, P<0.001)$ lower in the period after telemedicine rounds were introduced. The mortality rates per 100 person-years $(95 \% \mathrm{CI})$ were 11.04 versus 7.99 in the compared groups, respectively, with no significant increase in mortality during the months when telemedicine was performed.

Conclusion: Patients received appropriate care in a timely manner, with telemedicine implementation, and such measures did not lead to suboptimal healthcare outcomes. Telemedicine was a successful tool for physician oversight under movement control measures implemented during the COVID-19 pandemic and may continue to prove useful in the 'new normal' era of healthcare delivery for HD patients in community-based dialysis centres, operated by the National Kidney Foundation in Singapore.
\end{abstract}

Ann Acad Med Singap 2020;49:756-63

Keywords: Healthcare outcomes healthcare system, National Kidney Foundation, SARS-CoV2, telemedicine rounds

\section{Introduction}

Pandemics and natural adversities challenge healthcare delivery systems. The traditional model of care in Singapore's community-based haemodialysis centres has been nephrologist-led, with monthly physical rounds by nephrologists. During these rounds, patient care issues including haemodialysis parameters, metabolic disease management, anaemia control, iron supplementation, blood pressure regulation and multiple chronic diseaserelated management are optimised. Telemedicine was adopted as a new standard of care by the National
Kidney Foundation (NKF) during the COVID-19 pandemic. NKF is an organisation that is at the forefront of providing community-based dialysis treatment, with commitment to journey with the patients during every step of their end stage renal disease (ESRD) care. Telemedicine by NKF was set up in concurrence with the Singapore Ministry of Health (MOH) directives on movement restrictions of healthcare workers across different institutions, following the rapid escalation of community COVID-19 transmission cases. ${ }^{1,2}$ Details of the swift implementation of telemedicine service in NKF dialysis

\footnotetext{
'Medical Services Department, National Kidney Foundation, Singapore

${ }^{2}$ Department of Medicine, National University Hospital, Singapore

Address for Correspondence: Dr Tripti Singh/ Dr Behram Ali Khan, Medical Services Department, National Kidney Foundation, 81 Kim Keat Road, Singapore 328836 .

Emails: singh.tripti@nkfs.org; behram.ak@nkfs.org
} 
centres (DCs) and its challenges have been previously described by Ngoh et al. ${ }^{3}$

As part of this new model of tele-haemodialysis, virtual patient rounds were conducted via video-conferencing with the nephrologist (if the physician was based primarily in a hospital), while patients and dialysis nursing staff remained physically at dialysis centres. Laboratory and dialysis reports were reviewed on an electronic medical record and progress notes were electronically logged. Other models of telecare have been described in remote communities in Canada and Australia, such as tele-case reviews with multidisciplinary teams. ${ }^{4}$ Although reports described no difference in health condition or care utilisation with these methods, the rapid transition to telemedicine in Singapore has raised some concerns. Here we compare medical outcomes in our HD cohort before and after telemedicine was introduced.

\section{Methods}

This was a retrospective analysis of clinical audit data from NKF DCs. Eleven of 37 DCs were excluded from the analysis as they saw the continuation of physical physician rounds, because the physicians involved did not have any hospital duties. Twenty-six DCs accounting for 2590 patients were included in the analysis. The 2 periods chosen for comparison were that of preintroduction of telemedicine rounds-1 November 2019 till 31 January 2020 - and post-introduction of telemedicine rounds-1 February till 30 April 2020. Hospitalisation and mortality rates were chosen, as these represent a culmination of various patient care indices translating into measurable hard outcomes.

The various parameters assessed were expressed as a mean with standard deviation. The matched-pairs t-test was used to compare pre-and post-introduction of telemedicine rounds. All analyses were performed with PASW Statistics (Version 18, SPSS, Chicago).

Data was presented as a mean (SD) or median (IQR) for continuous variables (normal and non-normal distributions, respectively) and $\mathrm{n}(\%)$ for categorical variables. Crude hospitalisation rates per 1,000 HD patients were calculated by dividing the number of hospital admissions by the total number of HD patients at the end of each time period. Hospitalisation rates per person-year were derived by dividing the hospital admissions by the total person-years in each time period. 95\% confidence intervals were derived using Byar approximation of Poisson regression. Negative binomial regression model correcting for over-dispersion was used to compare the incidence rate ratios (IRR) between the 2 time periods after adjusting for case-mix.
Crude death rates (expressed in \% for each quarter) were calculated by dividing total deaths by the total HD patients at the end of each time period. Absolute mortality rates were calculated per 100 patient-years of follow-up with $95 \%$ confidence intervals and comparisons made using Mid P Exact test. $P$ values $<0.05$ were considered statistically significant.

\section{Results}

\section{Patient characteristics}

Overall, there were 2589 and 2590 patients available for analysis in pre- and post-introduction of telemedicine rounds, respectively. Table 1 shows the 2 comparison groups' characteristics. Despite patient flux related to change of modality of renal replacement therapy to and from peritoneal dialysis, or kidney transplantation, or death, the number of patients between pre- and postintroduction of telemedicine rounds remained relatively stable. There were also no significant differences in the demographics between the HD patient cohorts during the 2 time frames (Table 1).

About $57 \%$ of the patients had diabetic kidney disease as the cause of their end-stage renal disease, and $69.8 \%$ were classified as having a high medical acuity status, defined by a set of NKF internal assessment criteria (Appendix 1), at the time of admission into the DCs. These 2 parameters remained statistically unchanged between pre- and post-introduction of telemedicine rounds.

Table 1. Characteristics of audit cohort

\begin{tabular}{|c|c|c|c|}
\hline & $\begin{array}{l}\text { Pre-Introduction } \\
\text { of Telemedicine } \\
\text { Rounds }\end{array}$ & $\begin{array}{l}\text { Post- } \\
\text { Introduction of } \\
\text { Telemedicine } \\
\text { Rounds }\end{array}$ & $P$ Value \\
\hline Number & 2589 & 2590 & \\
\hline Age $\left(\right.$ year) ${ }^{*}$ & $63.74 \pm 11.41$ & $63.87 \pm 11.41$ & 0.67 \\
\hline $\begin{array}{l}\text { Male gender, } \\
\text { no. }(\%)\end{array}$ & $1434(55.4 \%)$ & $1457(56.3 \%)$ & 0.53 \\
\hline $\begin{array}{l}\text { Chinese } \\
\text { ethnicity, } \\
\text { no. }(\%)\end{array}$ & $1496(57.8 \%)$ & $1511(58.3 \%)$ & 0.69 \\
\hline $\begin{array}{l}\text { Diabetes } \\
\text { mellitus as } \\
\text { aetiology of } \\
\text { ESRD, no. (\%) }\end{array}$ & $1476(57.0 \%)$ & $1474(56.9 \%)$ & 0.94 \\
\hline $\begin{array}{l}\text { High acuity } \\
\text { status, }{ }^{\dagger} \text { no. }(\%)\end{array}$ & $1806(69.8 \%)$ & $1813(70.0 \%)$ & 0.85 \\
\hline
\end{tabular}

ESRD: End-stage renal disease

*Values are expressed as means \pm standard deviation.

${ }^{\dagger}$ High acuity status is defined by NKF internal assessment criteria (Appendix 1). 
Appendix 1

\begin{tabular}{|c|c|c|c|c|c|}
\hline \multicolumn{2}{|c|}{ MEDICAL ASSESSMENTS } & \multicolumn{4}{|c|}{ NAME OF DOCTOR / NURSE : } \\
\hline \multicolumn{2}{|c|}{ [A] Coronary Artery Disease } & Yes & No & Score & Remarks \\
\hline \multicolumn{6}{|c|}{ Recent Myocardial Infarct (<3mths) } \\
\hline \multicolumn{6}{|c|}{ Myocardial Infarct (>3 mths) } \\
\hline \multicolumn{6}{|c|}{ Ischaemic Heart Disease including Angina, Coronary Artery Disease } \\
\hline \multicolumn{6}{|c|}{ Low Ejection Fraction <25\% (If 'Yes', please indicate date Ejection Fraction reported: } \\
\hline \multicolumn{6}{|c|}{ Uncontrolled Cardiac Arrhythmias } \\
\hline \multicolumn{6}{|c|}{ [ B ] Bleeding Risk } \\
\hline \multicolumn{6}{|c|}{ Dissecting Aneurysm } \\
\hline \multicolumn{6}{|c|}{ Abdominal Aortic Aneurysm } \\
\hline \multicolumn{6}{|c|}{ Coagulation Abnormality } \\
\hline \multicolumn{6}{|c|}{ Others: E.g. Bleeding GIT, on anti-coagulant etc } \\
\hline \multicolumn{6}{|c|}{ [ C ] Malignancy } \\
\hline \multicolumn{6}{|c|}{$\begin{array}{l}\text { Advance malignancy with metastasis } \\
\text { Any carcinoma }\end{array}$} \\
\hline \multicolumn{6}{|l|}{ Any carcinoma } \\
\hline \multirow{2}{*}{\multicolumn{6}{|c|}{ [ D ] Advanced Organ Failure }} \\
\hline \multicolumn{3}{|c|}{ End Stage Liver Cirrhosis } & & & \\
\hline \multicolumn{6}{|c|}{ Chronic Obstructive Pulmonary Disease } \\
\hline \multicolumn{6}{|l|}{ [E] Age } \\
\hline$<50$ years $(0 p$ & ooint) & & & & \\
\hline $51-60$ years $(1$ & point) & & & & \\
\hline $61-70$ years $(2$ & points) & & & & \\
\hline$>71$ years $(3 \mathrm{pc}$ & oints) & & & & \\
\hline [F]CVA & & & & & \\
\hline Any CVA & & & & & \\
\hline $\begin{array}{l}\text { [ G ] Unstable } \\
\text { (symptoms pre }\end{array}$ & $\begin{array}{l}\text { During Dialysis } \\
\text { esent in } 1 \text { out of } 3 \text { dialysis sessions in the last } 3 \text { dialysis sessions) }\end{array}$ & & & & \\
\hline Persistent Pro & oblematic Intradialytic Hypotension with symptoms & & & & \\
\hline $\begin{array}{l}\text { Others (e.g Ur } \\
\text { Please indicat } \\
\text { listed above }\end{array}$ & $\begin{array}{l}\text { ncontrolled Hypertension [180/110] / SOB / Chest pain ) } \\
\text { te under Remarks column if patient develop symptoms which are not }\end{array}$ & & & & \\
\hline SCORE FOR M & Total of Score $[A]$ to $[G]$ & & & & \\
\hline FUNCTIONAL & DEPENDENCY & & & & \\
\hline [ H ] Karnofsk & sy Scoring (K Score) & Scale & K Score & NKF Score & Remarks \\
\hline $\begin{array}{l}\text { You can rate b } \\
10 \text { to } 49\end{array}$ & between 1 to 100 for Karnofsky Score under K Score; for NKF score, indica & te '1' if & Score is & $\geq 80 ; 2^{\prime}$ if $K S c$ & re is 50 to 79 ; ' 3 ' if $K$ Score is \\
\hline & Normal no complaints; no sign \& symptoms of disease. & 100 & & & Able to carry on normal activity \\
\hline Independent & Able to carry on normal activity; minor signs or symptoms of disease. & 90 & & & and to work; no special care \\
\hline & Normal activity with effort; some signs or symptoms of disease. & 80 & & & \\
\hline & Cares for self; unable to carry on normal activity or to do active work & 70 & & & Unable to work; able to live at \\
\hline $\begin{array}{l}\text { Limited } \\
\text { Mobility }\end{array}$ & Requires occasional assistance, but able to care for most of personal needs. & 60 & & & $\begin{array}{l}\text { home and care for most } \\
\text { personal needs; varying amount }\end{array}$ \\
\hline & Requires considerable assistance and frequent medical care & 50 & & & of assistance needed. \\
\hline & Disabled; requires special care and assistance. & 40 & & & \\
\hline Non-ambulant & Severely disabled; hospital admission is indicated although death not imminent & 30 & & & $\begin{array}{l}\text { Unable to care for self; requires } \\
\text { equivalent of institutional or }\end{array}$ \\
\hline Patients & Very sick; hospital admission necessary; active supportive treatment necessary. & 20 & & & $\begin{array}{l}\text { hospital care; disease may be } \\
\text { progressing rapidly. }\end{array}$ \\
\hline & Moribund; fatal processes progressing rapidly. & 10 & & & \\
\hline [ I] Details of & Mobility (Use Limited Mobility to indicate assistance needed) & Yes & No & Score & Remarks \\
\hline Amputee (Spe & ecify details): & & & & \\
\hline Wheelchair Bc & ound & & & & \\
\hline [J] Others & & Yes & No & Score & Remarks \\
\hline Visual Impairn & ment & & & & \\
\hline Audio Impairn & ment & & & & \\
\hline Neurological I & Impairment ( Eg. Dementia, Retardation, Epilepsy etc) & & & & \\
\hline Problematic V & Jascular Access & & & & \\
\hline SCORE FOR FU & JNCTIONAL DEPENDENCY & & & & \\
\hline TOTAL MEDIC & CAL \& FUNCTIONAL DEPENDENCY SCORE ( Total of Score $[\mathrm{A}]$ to $[\mathrm{J}]$ ) & & & & \\
\hline
\end{tabular}




\section{Hospitalisation rates}

The crude hospitalisation rate was significantly lower post-introduction of telemedicine (630 per 1000 patientyears versus 541 per 1000 patient-years, $P<0.001)$. Total number of hospital admissions across 26 dialysis centres in the 2 study time frames were 1,572 and 1,379, respectively, excluding 67 and 46 elective admissions in the respective pre- and post-telemedicine rounding datasets. The number of patients excluded due to missing data were relatively low in both groups: 4 and 5 patients, respectively, had missing discharge date (they remained hospitalised at the end of the period being reviewed), while a further 3 and 4 patients, respectively, had missing diagnostic codes (Table 2).

The hospitalisation rate per person-year was significantly lower post-introduction of telemedicine (pre-introduction of telemedicine rounds: 22.11 (95\% CI: 21.74-22.48) versus post-introduction of telemedicine rounds: 16.31 (95\% CI: 16.0-16.64), $P<0.001)$. Similarly, after adjustment for case mix (adjusted for age, race, gender and ESRD cause; per person-year; IRR based on negative binomial regression models), the hospitalisation incidence was $13.9 \%$ (95\% CI: $5.6 \%-21.5 \%, P<0.001)$ lower in the period after telemedicine rounds were introduced (Table 3 ).

Table 2. Hospitalisation rates for the 26 dialysis centres comparing pre- and post-introduction of telemedicine rounds

\begin{tabular}{|c|c|c|}
\hline & $\begin{array}{c}\text { Pre-Introduction } \\
\text { of Telemedicine } \\
\text { Rounds }\end{array}$ & $\begin{array}{c}\text { Post-Introduction } \\
\text { of Telemedicine } \\
\text { Rounds }\end{array}$ \\
\hline Duration & $\begin{array}{c}1 \text { November 2019-31 } \\
\text { January } 2020\end{array}$ & $\begin{array}{l}1 \text { February } 2020- \\
30 \text { April } 2020\end{array}$ \\
\hline $\begin{array}{l}\text { Total hospital } \\
\text { admissions } \\
\text { (after exclusions) }\end{array}$ & 1572 & 1379 \\
\hline $\begin{array}{l}\text { Exclusions (missing } \\
\text { discharge date) }\end{array}$ & 4 & 5 \\
\hline $\begin{array}{l}\text { Exclusions (missing } \\
\text { diagnostic code) }\end{array}$ & 3 & 4 \\
\hline $\begin{array}{l}\text { Exclusions (elective } \\
\text { admissions) }\end{array}$ & 67 & 46 \\
\hline $\begin{array}{l}\text { Total prevalent HD } \\
\text { patients }\end{array}$ & $\begin{array}{c}2493 \\
\text { (as of } 31 \text { January } \\
2020)\end{array}$ & $\begin{array}{c}2549 \\
\text { (as of } 30 \text { April } \\
2020 \text { ) }\end{array}$ \\
\hline $\begin{array}{l}\text { Crude hospitalisation } \\
\text { rate per } 1000 \mathrm{HD} \\
\text { patients }(95 \% \mathrm{CI})\end{array}$ & $\begin{array}{c}630.6 \\
(611.4-649.3)\end{array}$ & $\begin{array}{c}541.00 \\
(521.6-560.3)\end{array}$ \\
\hline
\end{tabular}

CI: Confidence interval; HD: Haemodialysis
The commonest causes of hospitalisation among HD patients were vascular access dysfunction, infections, and cardiovascular disease. There was no statistically significant difference in overall hospitalisation rates in these subgroups between the 2 time frames. Hospitalisations related to vascular access dysfunction were $25.3 \%$ and $27.8 \%$, respectively, in both the time periods. Respiratory diseases and infection-related hospitalisations at $21.2 \%$ versus $20.2 \% \quad(P=0.022)$ and cardiovascular disease-related hospitalisations at $25.5 \%$ versus $20.9 \%(P<0.001)$, respectively, were significantly decreased in the post-telemedicine time period (Table 4). However, dialysis treatment-related hospitalisation rates, which include hospitalisation due to volume overload, severe hypotension, missed haemodialysis, pyrogenic reaction and hospitalisation for change in renal replacement therapy modality, demonstrated a statistically significant increase during the post telemedicine period (Table 4).

\section{Mortality outcomes}

There were 69 versus 49 deaths during the pre- and post-telemedicine time frames, with a crude mortality rate of $2.77 \%$ versus $1.92 \%$, respectively (95\% CI). The mortality rate per 100 person-years (95\% CI) were 11.04 versus 7.99 in the compared groups respectively, with no significant increase in mortality during the months when telemedicine was performed.

\section{Discussion}

The immunocompromised nature of HD patients is well documented in the literature, with Italian and Spanish studies both reporting COVID-19 mortality rates in infected HD patients exceeding $25 \% .{ }^{5,6} \mathrm{NKF}$ has adopted precautionary measures and protocols during the COVID-19 pandemic to minimise the spread of the infection.

These include:

a) Pre-dialysis enhanced screening with temperature measurement and declaration form (which includes travel history, health declaration of self and family) at entry.

b) Compulsory surgical mask to everyone (patients, accompanying care givers and all DC staff) throughout the dialysis session and while inside the DC.

c) Early referral to hospital/polyclinics for all with temperature more than 37.5 degree Celsius and those with acute respiratory symptoms.

d) Enhanced infection control measures in DC, which include frequent hand sanitation, early reporting of fevers or new symptoms to the doctors, better access 
Table 3. Hospitalisation rate and incidence rate ratio comparisons between pre- and post-introduction of telemedicine rounds

\begin{tabular}{|c|c|c|c|}
\hline & $\begin{array}{l}\text { Pre-Introduction of } \\
\text { Telemedicine Rounds }\end{array}$ & $\begin{array}{l}\text { Post-Introduction of } \\
\text { Telemedicine Rounds }\end{array}$ & $P$ Value \\
\hline Hospital admissions/no. at risk & $1572 / 2589$ & $1379 / 2590$ & \\
\hline $\begin{array}{l}\text { Hospitalisation rate per person year } \\
(95 \% \mathrm{CI})\end{array}$ & $\begin{array}{c}2.68 \\
(2.55-2.81)\end{array}$ & $\begin{array}{c}2.35 \\
(2.23-2.48)\end{array}$ & 0.002 \\
\hline Mean length of stay (days) & 8.79 days & 7.26 days & 0.018 \\
\hline $\begin{array}{l}\text { Total hospital days per person-year } \\
(95 \% \mathrm{CI})\end{array}$ & $22.11(21.74-22.48)$ & $16.31(16.0-16.64)$ & $<0.001$ \\
\hline Unadjusted IRR (with 95\% CI) & 1 (reference) & $0.867(0.791-0.951)$ & 0.002 \\
\hline Case mix ${ }^{*}$ adjusted IRR (with $95 \%$ CI) & 1 (reference) & $0.861(0.785-0.944)$ & 0.001 \\
\hline
\end{tabular}

CI: Confidence interval; IRR: Incidence rate ratio, based on negative binomial regression models.

* Adjusted for age, race, gender and ESRD cause.

Table 4. Reasons for hospitalisation

\begin{tabular}{|c|c|c|c|}
\hline Reason for Referral & $\begin{array}{l}\text { Pre-Telemedicine } \\
\text { Number }(\%)\end{array}$ & $\begin{array}{l}\text { Post-Telemedicine } \\
\text { Number }(\%)\end{array}$ & $P$ Value \\
\hline Vascular access-related & $397(25.3)$ & $384(27.8)$ & 0.56 \\
\hline Cardiovascular disease $^{*}$ & $401(25.5)$ & $288(20.9)$ & $<0.001$ \\
\hline Respiratory disease and infections & $334(21.2)$ & $279(20.2)$ & 0.022 \\
\hline Dialysis treatment-related & $67(4.3)$ & $105(7.6)$ & 0.023 \\
\hline Falls and trauma & $103(6.6)$ & $77(5.6)$ & 0.05 \\
\hline Others ${ }^{\dagger}$ & $137(8.7)$ & $140(10.2)$ & 0.75 \\
\hline Total & 1572 & 1379 & \\
\hline
\end{tabular}

"Includes coronary artery disease, other cardiac diseases, cerebrovascular disease and peripheral vascular disease.

$\dagger$ Includes endocrine, haematological, malignancy, genitourinary, dermatological, ear, nose or throat disorders, drug reactions, psychiatric disorders, rehabilitation requirements, obstetric or gynaecological disorders.

to infection control team for any queries, increased ventilation of the premises, frequent cleaning of all surfaces in patient care areas, enhanced sterilisation protocols for haemodialysis machines, including hot rinsing in the morning and chemical disinfection after each session.

e) Segregation of DC staff with their allocated patients in each shift, with no mixing or changing of shifts for patients and DC staff, unless absolutely necessary.

f) Social distancing measures at work.

Electronic medical record was already in place at NKF when the decision to start virtual rounds in dialysis centres was implemented in the first week of February 2020. The aim was to mitigate the risk of COVID-19 spread with cessation of in-person dialysis rounds and switching to teleconsultation.

All rounding physicians already had remote accessenabled tablet computers (iPads). As part of the teleconsultation practice, the physicians were able to communicate with the dialysis nurses for all patients via phone or Microsoft Teams platform to conduct their rounds, with easy access to electronic medical record containing all necessary dialysis treatment records. These teleconsultation rounds were conducted on a set monthly basis and on additional ad hoc basis if needed. 
With such a teleconsultation method, one concern was the difficulty in assessing the patient's fluid status and the resultant estimated dry weight titration. Trained NKF clinical nurse managers, who underwent proficiency assessment in fluid assessment for patients, helped mitigate these concerns for the rounding physicians.

Telemedicine was swiftly implemented to limit patient-physician interactions. These measures have been largely successful, as to-date, there have been only 3 reported cases of COVID-19 in the NKF HD cohort. The potential advantages and challenges of using telemedicine during the COVID-19 pandemic have been discussed elsewhere. ${ }^{7,8}$

\section{Difference in hospitalisation and mortality rates}

Mortality rates were unchanged by the introduction of telemedicine. Unexpectedly, we found that the overall hospitalisation rates werelowerin theperiodafterintroduction of telemedicine rounds (Tables 5 and 6). There are several plausible reasons for this. Firstly, hospitals have scaled back on non-urgent investigations and elective surgeries in preparation for the COVID-19 pandemic. Secondly, the triage protocol and enhanced infection control measures put in place during this pandemic likely helped. All patients in NKF HD centres were actively screened during each dialysis session for signs and symptoms suggestive of COVID-19 infection, including temperature monitoring. Patients who failed triage were referred to the local primary care physicians in polyclinics for further management where COVID-19 diagnostic swabs were performed, if indicated. This led to more patients being screened, undergoing diagnostic testing and receiving treatment in outpatient settings, effectively reducing hospital visits.

In addition, the closure of food and recreation outlets during government-mandated 'circuit breaker phase 1', which was implemented between 7 April 2020 and 1 June 2020, coincided with the time period under review. HD patients had better salt and fluid control as a result of these social restrictions, which led to better blood pressure and volume control. This would explain the significantly reduced volume of cardiac-related admissions after the introduction of telemedicine rounds. To our knowledge, this phenomenon has not been reported elsewhere. Finally, a reduction in traumatic injuries from road traffic accidents or street crimes during this period of movement control measures may have contributed as well. However, reports have demonstrated an uptick of domestic violence and psychological affects during these periods of prolonged social isolation, ${ }^{9-11}$ the effects of which cannot be commented on for the purpose of this analysis.

\section{Hospitalisations related to vascular access dysfunction}

There was no significant difference noted in the number of hospitalisations related to vascular access dysfunction during both the time frames under review. This indicates that vascular access surveillance can be safely carried out with telemedicine model of care. Patients with urgent vascular needs were managed by telemedicine consultation, followed by day surgery procedures. Day surgery admissions were not captured as hospitalisations in the clinical audit data being reported.

\section{Hospitalisations related to dialysis treatment complications}

Hospitalisations related to dialysis treatment complications increased after the introduction of telemedicine rounds. This is more likely a reflection of changing healthcare policies in response to the COVID-19 situation and altered workflows. ${ }^{12}$ The majority $(>60 \%)$ of these referrals were initiated by the DCs for reasons including fever, which was more stringently defined as a reading above 37.5 degree Celsius, with the enhanced screening measures implemented. These patients may not have been sent to the hospital in the pre-pandemic era, due to absence of such infection control measures then. A significant number of these patients were labelled as dialysis-related admissions as they had no positive bacteriology during hospital admission, but required dialysis for fluid overload, having missed a planned community-based dialysis session. Other reasons for cases being categorised as dialysis treatmentrelated hospitalisation in our analysis were those with hypotension, hypertensive urgency, shortness of breath with fluid overload secondary to baseline cardiac dysfunction or arrhythmias, asthma exacerbation and fluid indiscretion. These scenarios leading to increased hospitalisation can be partially explained by the change in workflows prohibiting extra HD sessions, which was implemented once again as an infection control protocol to avoid cross-contamination between different HD shifts and to aid in contact tracing of potential COVID-19 cases.

Seasonal variation is noticed in some countries when respiratory and cardiovascular illnesses are common in winter, and gastrointestinal infectious illnesses predominate in summer. However, as Singapore is situated in the tropics, the seasonal variation is minimal and no diurnal peaks are seen in disease distribution. Our study 
Table 5. Mortality rates for the 26 dialysis centres during the 2 time periods

\begin{tabular}{|c|c|c|c|}
\hline & Pre-Telemedicine & Post-Telemedicine & $P$ Value \\
\hline Duration & 1 November 2019-31 January 2020 & 1 February 2020-30 April 2020 & \\
\hline Total number of deaths & 69 & 49 & \\
\hline Total prevalent HD patients & $\begin{array}{c}2493 \\
\text { (as of 31 January 2020) }\end{array}$ & $\begin{array}{c}2549 \\
\text { (as of 30 April 2020) }\end{array}$ & \\
\hline $\begin{array}{l}\text { Crude mortality rate (\%) per quarter } \\
(95 \% \mathrm{CI})\end{array}$ & $\begin{array}{c}2.77 \% \\
(2.18-3.47)\end{array}$ & $\begin{array}{c}1.92 \% \\
(1.44-2.51)\end{array}$ & \\
\hline $\begin{array}{l}\text { Mortality rate per } 100 \text { person-years } \\
(95 \% \mathrm{CI})\end{array}$ & $\begin{array}{c}11.04 \\
(8.59-13.97)\end{array}$ & $\begin{array}{c}7.99 \\
(5.91-10.56)\end{array}$ & 0.08 \\
\hline
\end{tabular}

CI: Confidence interval; HD: Haemodialysis

Table 6. Causes of death

\begin{tabular}{|c|c|c|c|}
\hline Cause & $\begin{array}{l}\text { Pre-Telemedicine } \\
\text { Number (\%) }\end{array}$ & $\begin{array}{c}\text { Post-Telemedicine } \\
\text { Number (\%) }\end{array}$ & $P$ Value \\
\hline Cardiovascular disease ${ }^{*}$ & $32(46.4)$ & $21(42.9)$ & 0.71 \\
\hline Infections $^{\dagger}$ & $19(27.5)$ & $14(28.6)$ & 0.90 \\
\hline Malignancy & $3(4.3)$ & $3(6.1)$ & $\mathrm{Nil}$ \\
\hline Miscellaneous & $15(21.7)$ & $11(22.4)$ & 0.93 \\
\hline Total deaths & 69 & 49 & \\
\hline
\end{tabular}

*Includes coronary artery disease and cerebrovascular disease.

$\dagger$ Includes sepsis and pneumonia.

\$No $P$ value due to small sample.

Includes GI bleeding, head injury, hepatic failure, old age, pending death certificates, unknown (no death certificate), ESRD-NOS (not otherwise specified).

design was time-bound, which started with the beginning of the COVID-19 outbreak in February 2020. We do not expect any difference in seasonal variations, if the outbreak had occurred at a different time of the year.

The limitations of this analysis include reliance on clinical audit data with a short duration of follow-up. Our study compared 2 equal time periods before and after the beginning of the COVID-19 pandemic in Singapore. The interventions put in place during this time frame, including switching from in-person rounds to teleconsultation, was time-sensitive, and reverted back to in-person rounds with directions received via $\mathrm{MOH}$ circulars, as the number of COVID-19 cases in the community declined. This limits any increase in the duration of the study, which is time-barred to the period being reported here.
All the hospitalisation and mortality data have been extracted from electronic medical records and related data systems. Some potential errors in categorising patients, such as reasons for hospitalisations and the exact cause of death, were inevitable. There were also some data that could not be captured due to the nonavailability of death certificate and incorrect coding from the hospital's staff by merely mentioning that admission diagnosis as ESRD.

\section{Conclusion}

In conclusion, a model of healthcare delivery utilising telemedicine for the care of HD patients had similar outcomes in comparison to conventional in-person physician rounding. Telemedicine can be considered as a useful tool for physician oversight in the setting of a 
community-based HD centre. This is especially pertinent in situations where access to such centres may have to be limited, such as in an infectious disease outbreak. It may even be considered as an innovation in the delivery of modern healthcare, as part of the 'new normal' for the future. Added advantages, such as decreased commuting time for physicians, may greatly help in increasing work efficiency and aid in capacity-building at times of strains being felt by the healthcare system during pandemics. Further studies assessing other aspects of telemedicine models of HD care delivery, including patients' psychological acceptance of remote physician oversight and detailed cost-benefit analysis to the healthcare system, are required.

\section{REFERENCES}

1. Ministry of Health, Singapore. Guidance on Cross-Institutional Movement. MOH Cir. No. 102-2020. 20 April 2020.

2. Ministry of Health, Singapore. Guidance on Movement of HCW. MOH Cir. No. 60-2020. Updated: Guidance on Movement of HCW Patients and Visitors. 27 February 2020.

3. Ngoh CLY, Wong WK, Cheang CHL, et al. Rapid Transition to a Telemedicine Service at Singapore Community Dialysis Centers During Covid-19. NEJM Catalyst Innovations in Care Delivery.
Available at: https://catalyst.nejm.org/doi/full/10.1056/cat.20.0145. Accessed on 28 May 2020.

4. Sicotte C, Moqadem K, Vasilevsky M, et al. Use of telemedicine for haemodialysis in very remote areas: the Canadian First Nations. J Telemed Telecare 2011;17:146-9.

5. Alberici F, Delbarba E, Manenti C, et al. A report from the Brescia Renal COVID Task Force on the clinical characteristics and short-term outcome of hemodialysis patients with SARS-CoV-2 infection. Kidney Int 2020;98:20-6.

6. Goicoechea M, Cámara LAS, Macías M, et al. COVID-19: clinical course and outcomes of 36 hemodialysis patients in Spain. Kidney Int 2020;98:27-34.

7. Lurie N, Carr BG. The role of telehealth in the medical response to disasters. JAMA Intern Med 2018;178:745-6.

8. Hollander JE, Carr BG. Virtually perfect? Telemedicine for Covid-19. N Engl J Med 2020;382:1679-81.

9. Sacco MA, Caputo F, Ricci P, et al. The impact of the Covid-19 pandemic on domestic violence: The dark side of home isolation during quarantine. Med Leg J 2020;88:71-3.

10. Mengin A,Allé MC, Rolling J, et al. Conséquences psychopathologiques du confinement [Psychopathological consequences of confinement]. Encephale 2020;46:S43-52.

11. Ho CSH, Chee CY, Ho RCM. Mental health strategies to combat the psychological impact of coronavirus disease 2019 (COVID-19) beyond paranoia and panic. Ann Acad Med Singap 2020;49:155-60.

12. Lim TK. The facts, fallacies and uncertainties about coronavirus disease 2019 (COVID-19). Ann Acad Med Singap 2020;49:343-5. 